\title{
The Research on Rolling Motion Model of the SWATH-USV Based on System Identification
}

\author{
Xue $\mathrm{Cao}^{1, \text { a }}$, Man Liü, ${ }^{2, b}$, Song-lin Yang ${ }^{3, \mathrm{c}}$ \\ ${ }^{1,2,3}$ School of Naval Architecture and ocean engineering, Jiangsu University of Science and \\ Technology, Zhenjiang ,Jiangsu,China \\ a1073881169@qq.com, b941376267@qq.com, cysl560516@vip.163.com
}

Keywords: SWATH-USV; Ship model test; System identification; Roll motion model

\begin{abstract}
In this paper, a SWATH USV is taken as the research object, a series of static roll attenuation tests are carried out under different draft and different initial roll angles. By changing the composition of the restoring moment and damping, 4 mathematical models of roll attenuation motion system identification are established. And based on system identification theory and a variety of optimization algorithms programming ideas, C\# language is used to rewrite the system identification software including genetic algorithm and particle swarm algorithm. The identification and analysis of the rolling data of unmanned craft are carried out, and a better mathematical model of rolling motion is obtained by identification and calculation, and the variation of identification parameters under different draught and inclination angles is compared. The relative error between the experimental value and the forecast value predicted by the software, in good agreement with experimental values, proved the reliability of the system identification method, can forecast the future of unmanned navigation parameters and motion attitude.
\end{abstract}

\section{Introduction}

The SWATH-USV is composed of the left and right submerged body, pillar and upper structures far from the waterline ${ }^{[1]}$, which with excellent seakeeping performance. Compared with conventional mono hull ship, its roll angle is smaller, only 2.5 degrees -3 degrees. But its natural cycle is much greater ${ }^{[2]}$.

It is easy to produce large rolling motion when ships navigate in waves, and the large rolling motion exhibits complex nonlinearity ${ }^{[3]}$.When the SWATH navigates in oblique waves, it is easy to occur the coupling phenomenon of rolling and pitching ${ }^{[4]}$,therefore, it is very important to study and predict the roll motion of a SWATH ${ }^{[5]}$.Model test, however, is an effective means of studying rolling motion,in addition,the system identification method which is based on the optimal design has been applied to rolling motion tests of monohull ship model and trimaran model ${ }^{[6-7]}$.

In this paper, the model test of rolling motion in still water of a SWATH-USV is carried out, then a mathematical model of linear and nonlinear roll attenuation motion modes are established according to the principle of dynamic balance in rolling.After that,the relevant design variables and objective functions are determined respectively, and the genetic and particle swarm optimization algorithm system identification program is written which is based on C\#.The rolling test data are identified and analyzed through the program,the results show that the error between the test values and the predicted values is smaller which can prove the reliability of this system identification software. 


\section{System Identification}

System identification is a branch of modern control theory,it is based on the input and output data from known or measured system.It can determine a model that is equivalent to the system under test from a given set of model classes ${ }^{[8]}$. The three elements of system identification are data, model class and equivalence criterion.Among them, data is the basis of identification,which refers to the input data and output data of the system ${ }^{[9]}$; the model class is the range of the model to be identified,it refers to a collection of known system models;equivalence criterion is the objective of identification optimization, which refers to the identification criterion of system behavior similarity and system utility equivalence. The essence of identification is to select a model from a set of known model classes according to a certain criterion so that it can best fit the dynamic characteristics of the actual process ${ }^{[10]}$.

\section{Rolling test in still water}

\section{A. Test model}

In the rolling test, an optimized SWATH-USV model is selected and its main dimension is shown in Table 1:

Table 1. The main parameters of the optimized SWATH-USV

\begin{tabular}{cccccc}
\hline main dimension & value & Unit & main dimension & value & Unit \\
\hline Length $(\mathrm{L})$ & 1.26 & {$[\mathrm{~m}]$} & Design speed(Vs) & 5 & {$[\mathrm{kn}]$} \\
Breadth(B) & 0.6 & {$[\mathrm{~m}]$} & Hull spacing(Co) & 0.54 & {$[\mathrm{~m}]$} \\
Deep(D) & 0.37 & {$[\mathrm{~m}]$} & Length of submerged body(Lh) & 1.28 & {$[\mathrm{~m}]$} \\
Design draft(T) & 0.154 & {$[\mathrm{~m}]$} & Submerged body diameter(D1) & 0.128 & {$[\mathrm{~m}]$} \\
Design displacement $(\Delta)$ & 30 & {$[\mathrm{~kg}]$} & strut length(Ls) & 1.18 & {$[\mathrm{~m}]$} \\
Longitudinal spacing( $\left(\mathrm{S}_{1}\right)$ & 0.066 & {$[\mathrm{~m}]$} & Maximum width of strut(ts) & 0.538 & {$[\mathrm{~m}]$} \\
Frame spacing(S $)$ & 0.113 & {$[\mathrm{~m}]$} &
\end{tabular}

\section{B. The procedure of rolling test}

This rolling test in still water is carried out in the test tank and the specific test process is as follows:

(1)Test preparation: ship model with a length of 1.47 meters, inertial measuring instrument MTi-G ( six degrees of freedom motion of a ship can be measured), data transmission line, shore computer;

(2)In order to avoid the wall effect, the ship model is placed in the middle of the pool, and the ship model is positive floating by loading weights, so that the draft is at the designed waterline;

(3)Placing theinertial measuring instrument MTi-G at the center of gravity of the ship model horizontally and connecting the equipment with data lines;

(4)When the ship model is in the positive floating state, observing the data reader on the shore computer to read the roll angle of ship model,then Ensuring the initial roll angle is between \pm 0.2 degree by adjusting the MTi-G horizontal position;

(5)Giving the ship a desired roll angle manually, then clicking on the recorded data while releasing the ship model.When the ship model is restored to a positive state, stopping and saving the test data;

(6)Repeating step (5) to change the initial roll angle,when the ship model is in designed draft, the initial roll angle consists of four sets including $5^{\circ}, 10^{\circ}, 15^{\circ}$ and $20^{\circ}$;

(7)Then the rolling test is carried out of 1.1 and 0.9 times designed draft by adding or reducing ballast,then repeating the step (4) - (6),the test process is shown in figure 1: 


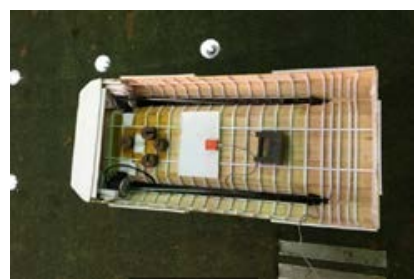

Fig. 1. Rolling attenuation test in still water of SWATH-USV

\section{Mathematical model of rolling identification}

According to the formula of hydrostatic moment balance:

$$
\ddot{\phi}+2 N \dot{\phi} / I_{x x}^{\prime}+W|\dot{\phi}| \dot{\phi} / I_{x x}^{\prime}+x \dot{\phi}^{3} / I_{x x}^{\prime}+D h \phi / I_{x x}^{\prime}=0
$$

The restoring moment in rolling equation are transformed in the form of the first,second,third power and the sine respectively, so as to find the rolling equation adapted to the ship model in this paper.

Identification Eq.1:

$$
\ddot{\phi}+2 N \dot{\phi} / I_{x x}^{\prime}+W|\dot{\phi}| \dot{\phi} / I_{x x}^{\prime}+x \phi^{3} / I_{x x}^{\prime}+C_{1} / I_{x x}^{\prime} \phi^{3}+C_{2} / I_{x x}^{\prime} \phi^{2}+C_{3} / I_{x x}^{\prime} \phi=0
$$

Design variables: $I_{x x}^{\prime}, N, W, x, C_{1}, C_{2}, C_{3}$, the range of design variables:

$$
\begin{aligned}
& I_{x x}^{\prime} \in[0,0.1], N \in[0,1], W \in[0,1], x \in[0,10], \\
& h \in[0,10], C_{1} \in[0,10], C_{2} \in[0,10], C_{3} \in[0,10]
\end{aligned}
$$

Identification Eq.2:

$$
\ddot{\phi}+2 N \dot{\phi} / I_{x x}^{\prime}+W|\dot{\phi}| \dot{\phi} / I_{x x}^{\prime}+x \phi^{3} / I_{x x}^{\prime}+D h \phi / I_{x x}^{\prime}=0
$$

Design variables: $I_{x x}^{\prime}, N, W, x, h$, the range of design variables:

$$
I_{x x}^{\prime} \in[0,0.1], N \in[0,1], W \in[0,1], x \in[0,10], h \in[0,10]
$$

Identification Eq.3:

$$
\ddot{\phi}+2 N \dot{\phi} / I_{x x}^{\prime}+W|\dot{\phi}| \dot{\phi} / I_{x x}^{\prime}+x \phi^{3} / I_{x x}^{\prime}+C_{1} / I_{x x}^{\prime}(\sin \phi)^{3}+C_{2} / I_{x x}^{\prime}(\sin \phi)^{2}+C_{3} / I_{x x}^{\prime} \sin \phi=0
$$

Design variables: $I_{x x}^{\prime}, N, W, x, C_{1}, C_{2}, C_{3}$, the range of design variables:

$$
\begin{aligned}
& I_{x x}^{\prime} \in[0,0.1], N \in[0,1], W \in[0,1], x \in[0,10], \\
& h \in[0,10], C_{1} \in[0,10], C_{2} \in[0,10], C_{3} \in[0,10]
\end{aligned}
$$

Identification Eq.4:

$$
\ddot{\phi}+2 N \dot{\phi} / I_{x x}^{\prime}+W|\dot{\phi}| \dot{\phi} / I_{x x}^{\prime}+x \phi^{3} / I_{x x}^{\prime}+C_{1} / I_{x x}^{\prime} d \sin \phi+C_{2} / I_{x x}^{\prime} \sin \phi=0
$$

Design variables: $I_{x x}^{\prime}, N, W, x, C_{1}, C_{2}$, the range of design variables:

$$
I_{x x}^{\prime} \in[0,0.1], N \in[0,1], W \in[0,1], x \in[0,10], h \in[0,10], C_{1} \in[0,10], C_{2} \in[0,10], C_{3} \in[0,10]
$$


In the four identification formulas, $\phi$ is the rolling angle; $\dot{\phi}$ is the angular speed of rolling ; $\ddot{\phi}$ is the angular acceleration of rolling; $I_{x x}^{\prime}$ is the total moment of inertia of rolling; $N$ is the linear damping coefficient of rolling; $w, x$ are nonlinear damping coefficients of rolling;C1,C2are the nonlinear restoring moment coefficients;C3is the linear restoring moment coefficient.

In the above four identification models, by measuring the angle of the test $K$, the criterion of error estimation at $K+1$ is as follows:

$$
\varepsilon_{k+1}=\dot{\phi}_{k+1}^{\prime}-\dot{\phi}_{k+1}
$$

$\dot{\phi}_{K+1}$ is the measurement of the $\mathrm{K}+1$ angular velocity; $\dot{\phi}_{K+1}^{\prime}$ is the value of the identification result.

Selecting the target function as shown in type 2.7,the smaller the value of the objective function, the better the formula fitting effect.

$$
F(x)=\left(\sqrt{1 / N \sum_{k=1}^{N}\left(\dot{\phi}_{K+1}^{\prime}-\dot{\phi}_{K+1}\right)^{2}}\right)^{-1}
$$

\section{Optimization calculation of rolling identification}

\section{A. The analysis of rolling test results}

To complete the rolling test of SWATH in still water, 3 tests are carried out for each initial roll angle, and the best set of experimental data was selected as valid data.Due to the man-made factors, there is some error in the test,Fig. 2 is the rolling angular velocity decay curve the conditions of the initial roll angle is 3 degrees, the draft is respectively 0.9 times design draft, design draft and 1.1 times design draft.It can be seen from the diagram that the roll angular velocity decay conforms to the rule, so the rolling test data is available.

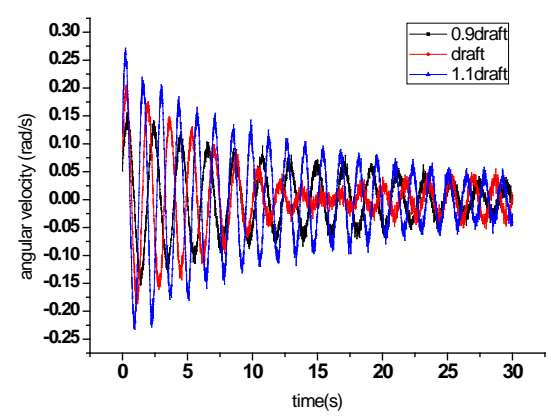

Fig. 2. Rolling angular velocity attenuation curve at different draft and initial roll angle of 3 degrees

As shown in Fig.2,in the three groups of different draft and the initial rolling angle of 3 degrees,the peak value of the roll angular velocity increases and the rolling period decreases with the draft increasing,it shows that the draft is larger, the seakeeping performance is better; Observing the red curve (design draft) in Fig.3.1,the decay rule is abnormal after 17 seconds,the reason may be that the wave disturbance caused by human factors makes the rolling motion more serious.In addition, the roll attenuation period of this model is longer than that of a single hull ship,firstly,it is 
due to the ship type,rolling motion is accompanied by pitching and the cycles of them are closed which resulting in a twist motion. The second reason is the layout factor, where the center of gravity of the ship model is on the high side due to ballast loading, so it needs improvement.Since the roll motion is affected after 15s, therefore, only the test data of the previous $15 \mathrm{~s}$ are taken.

\section{B. The scheme of rolling identification}

Conceptual design:the improved genetic algorithm and the particle swarm optimization algorithm are selected as the identification algorithm.

Mathematical model: identification formula 1.

Scheme 1:

Basic parameter settings of improved genetic algorithm: population size is 200,crossover probability is 0.8 , Mutation probability is 0.1 ; The calculation times is 1000 , Genetic factor is 0.05 , the evolutionary weights are $0.3,0.5,0.7$ and 0.9 , respectively.

\section{Scheme 2:}

Basic parameter settings of particle swarm optimization algorithm:population size is 200 , the variable weight are $0.4-0.9$, the maximum speed limits of particle flight are $0.11,0.13,0.15$ and 0.17 respectively, the calculation times is 1000 。

The test data consisted of 12 groups, which are drawn from the three draft states of the ship model, and the corresponding rolling angles are 5 degrees, 10 degrees, 15 degrees and 20 degrees respectively.Selecting the test data in the conditions of designed draft and the corresponding rolling angles are 5 degrees, 10 degrees, 15 degrees and 20 degrees.The test data are identified by scheme 1 and scheme 2 respectively,the value of the identification objective function is shown in Table 2:

Table 2. Identification calculation results of scheme 1

\begin{tabular}{|c|c|c|c|c|c|}
\hline \multicolumn{2}{|c|}{ Evolutionary weight } & 0.3 & 0.5 & 0.7 & 0.9 \\
\hline \multirow{4}{*}{$\begin{array}{c}0.9 \text { times } \\
\text { designed } \\
\text { draft }\end{array}$} & roll angle $5^{\circ}$ & 0.01738895 & 0.01738691 & 0.01738604 & 0.01738435 \\
\hline & roll angle $10^{\circ}$ & 0.02077764 & 0.02072318 & 0.02071295 & 0.02071804 \\
\hline & roll angle $15^{\circ}$ & 0.02103668 & 0.02103155 & 0.02103288 & 0.02103049 \\
\hline & roll angle $20^{\circ}$ & 0.02539442 & 0.02539193 & 0.02539155 & 0.02539095 \\
\hline \multirow{4}{*}{$\begin{array}{c}\text { designed } \\
\text { draft }\end{array}$} & roll angle $5^{\circ}$ & 0.01281120 & 0.01281116 & 0.01281118 & 0.01281121 \\
\hline & roll angle $10^{\circ}$ & 0.02045742 & 0.02044366 & 0.02347635 & 0.02044048 \\
\hline & roll angle $15^{\circ}$ & 0.01997139 & 0.02044048 & 0.01997051 & 0.01997051 \\
\hline & roll angle $20^{\circ}$ & 0.02210424 & 0.02210423 & 0.02210352 & 0.0234764 \\
\hline \multirow{4}{*}{$\begin{array}{c}1.1 \text { times } \\
\text { designed } \\
\text { draft }\end{array}$} & roll angle $5^{\circ}$ & 0.02319582 & 0.02482064 & 0.02340581 & 0.08010101 \\
\hline & roll angle $10^{\circ}$ & 0.05923744 & 0.01934433 & 0.01934354 & 0.01934116 \\
\hline & roll angle $15^{\circ}$ & 0.02482049 & 0.02018943 & 0.02018994 & 0.02018932 \\
\hline & roll angle $20^{\circ}$ & 0.02418359 & 0.02418412 & 0.02418446 & 0.02418314 \\
\hline
\end{tabular}


Table 3. Identification calculation results of scheme 2

\begin{tabular}{cccccc}
\hline $\begin{array}{c}\text { Maximum percentage of particle } \\
\text { flight speed limit }\end{array}$ & 0.11 & 0.13 & 0.15 & 0.17 \\
\hline \multirow{2}{*}{$\begin{array}{c}\text { roll angle 5 } \\
\text { designed draft }\end{array}$} & 0.01739260 & 0.01738919 & 0.01739195 & 0.01738916 \\
& roll angle 10 & 0.02140908 & 0.02116442 & 0.02115219 & 0.02115456 \\
& roll angle 15 & 0.02120734 & 0.02132037 & 0.02122287 & 0.02119557 \\
& roll angle 20 & 0.02687487 & 0.02628156 & 0.02594624 & 0.02567094 \\
& roll angle 5 & 0.01281165 & 0.01281195 & 0.01281254 & 0.01281179 \\
designed draft & roll angle 10 & 0.02082933 & 0.02082933 & 0.02084111 & 0.02083244 \\
& roll angle 15 & 0.02035625 & 0.02011385 & 0.02013282 & 0.02004665 \\
& roll angle 20 & 0.02231015 & 0.02228699 & 0.02244212 & 0.02230838 \\
& & & & & \\
1.1times & roll angle 5 & 0.08010124 & 0.08010845 & 0.08010178 & 0.08010147 \\
designed draft & roll angle 10 & 0.01959428 & 0.01960844 & 0.01961921 & 0.01956700 \\
& roll angle 15 & 0.02056346 & 0.02090378 & 0.02056140 & 0.02046167 \\
& roll angle 20 & 0.03192279 & 0.02462018 & 0.02494920 & 0.02466763 \\
\hline
\end{tabular}

In contrast to scheme one and scheme two,it can be found that the objective function value calculated by genetic algorithm is smaller than that calculated by particle swarm algorithm,that is to say the variance between the test value and the fitting value is small, and the fitting effect the of mathematical model is good.In the latter calculation, selecting the improved genetic algorithm, which has 200 population size, 0.8 crossover probability, 0.15 mutation probability, 0.05 genetic factor and 0.9 evolutionary weights.

Contrasting the 0.9 times design draft, design draft and 1.1 times draft three working conditions and choosing the different formulas corresponding to different draught, the result is shown in figure 2.2:

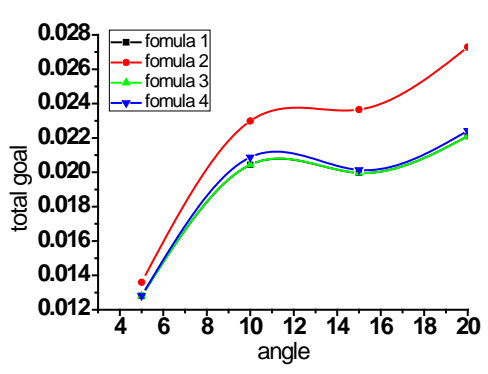

(a) 0.9 times designed draft

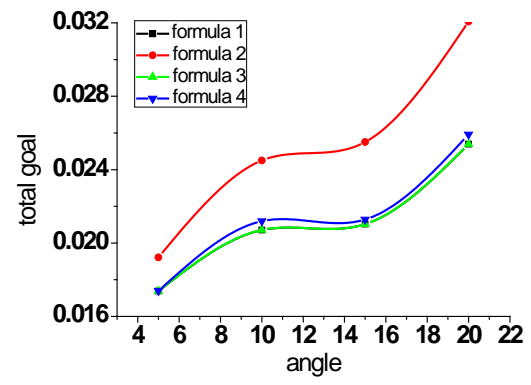

(b) Designed draft

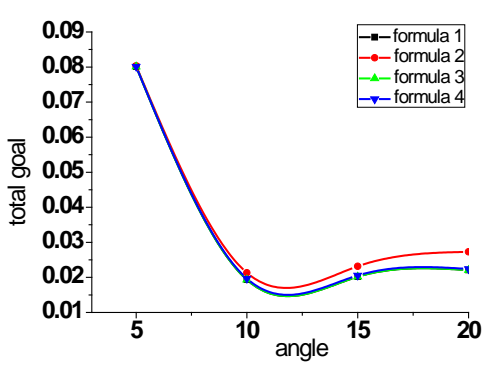

(c) 1.1 times designed draft

Fig. 3. Calculation results of three different draft and four identification formulas

As can be seen from Fig 3, under the three different drafts, the minimum objective function is calculated by Formula 1, which shows that formula 1 has the best fitting effect.Taking the design draft and the initial roll angle 5 degrees as an example, the target function value is 0.012811186 after identification, and the design variables are shown in Table 4: 
Table 4.The optimal values of each design variable are identified by Formula 1

\begin{tabular}{cccc}
\hline design variables & upper limit & lower limit & optimization value \\
\hline Total moment of inertia of rolling $I x$ & 0 & 0.1 & 0.09484457 \\
Linear damping moment coefficient of rolling $N x$ & 0 & 1 & 0.01397576 \\
Squared damping $w$ & 0 & 1 & 0.00125340 \\
Cubic damping $x$ & 0 & 10 & 1.14244500 \\
Coefficient of righting moment $C 1$ & 0 & 10 & 6.58768553 \\
Coefficient of righting moment $C 2$ & 0 & 10 & 0.00263590 \\
Coefficient of righting moment $C 3$ & 0 & 10 & 1.34854038 \\
\hline
\end{tabular}

The rolling motion model is as fllows:

$$
\begin{aligned}
& \ddot{\phi}+2 \times 0.013975755 \dot{\phi} / 0.094844567+0.001253404|\dot{\phi}| \dot{\phi} / 0.094844567 \\
& +1.142445001 \phi^{3} / 0.094844567+6.587685528 / 0.094844567 \phi^{3} \\
& +0.002635904 / 0.094844567 \phi^{2}+1.348540382 / 0.094844567 \phi=0
\end{aligned}
$$

In order to analyze the the error of test values and fitted values,puting the angular velocity test values and the fitting values identified by Formula 1 under the conditions of design draft and rolling angle of 5 degrees in the same figure, it is shown in Fig.4:

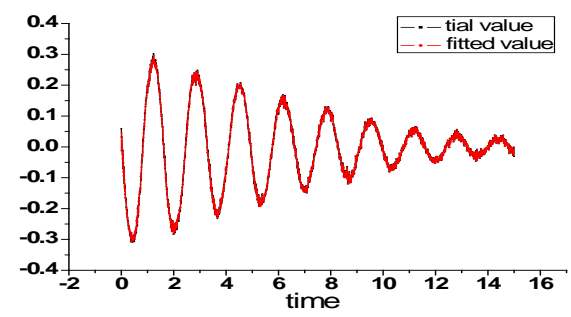

Fig 4. Fitting curve of test angular velocity and identification angular velocity

It can be seen from figure 3.3 that the fitting effect of the test angular velocity and the identification angular velocity is good, it can prove the reliability of identification software,therefore, the identification software can predict the roll angle speed at the next moment.

Total moment of inertia of rolling $I x$,the linear damping coefficient of rolling $\mathrm{N}$, the squared damping $w$,the cubic damping $x$ and the coefficients of restoring moment are obtained by identification calculation of rolling test data of 12 groups.Among them, the variation curve of each design variable with the initial rolling angle of three draft is shown in Fig. 4-5:

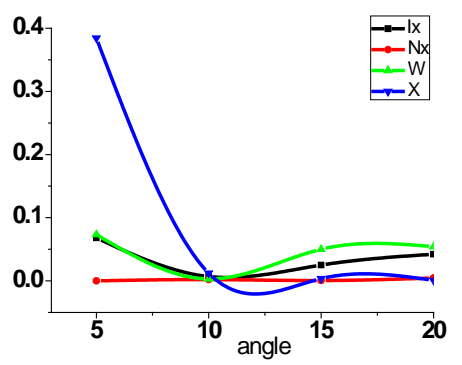

(a)

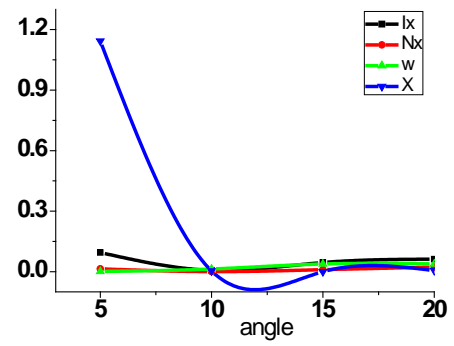

(b)

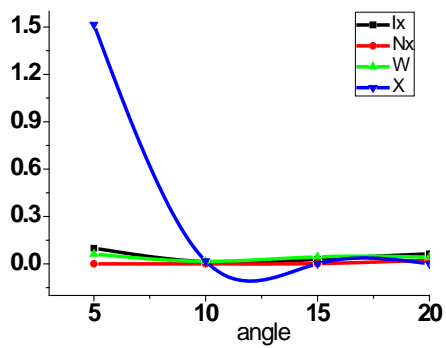

(c)

Fig.5. Curves of the moment coefficients of 0.9 times design draft(a),design draft(b) and1.1 times design $\operatorname{draft}(\mathrm{c})$ with the initial rolling angle 


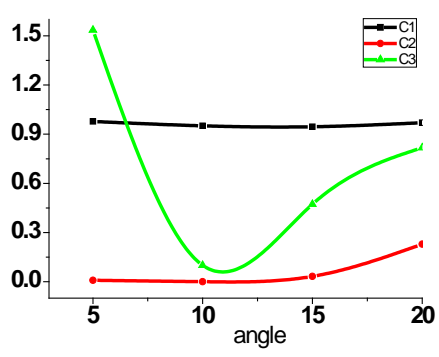

(a)

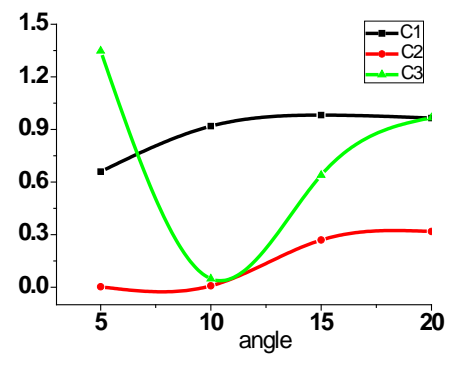

(b)

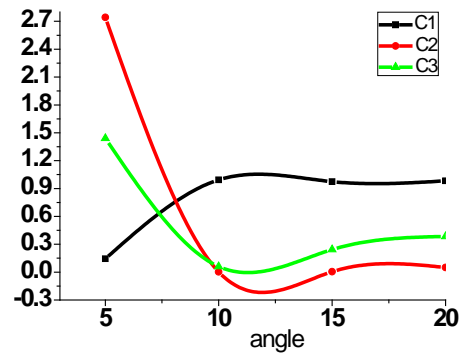

(c)

Fig.6. Curves of the moment coefficients of 0.9 times design draft(a),design draft(b) and1.1 times design $\operatorname{draft}(\mathrm{c})$ with the initial rolling angle

It can be seen from Fig. 4-5,the values of the torque coefficients vary with the draft and the initial rolling angle. When the initial rolling angle is simultaneous,the total moment of inertia of rolling increases with the increase of the draft,but the value of the linear damping coefficient is almost 0 ,it shows that there is no linear damping in the rolling motion of this ship model.The square damping is not obvious with the increase of draft ; The three square damping moment is larger at the angle of 5 degrees and increases with the increase of draft;With the increase of draft, the cubic restoring torque and linear restoring moment will decrease with the increase of draft.

As seen the Fig4 ,the damping coefficient of cubic rolling has the biggest change with the increase of rolling angle,at a small angle of 5 degrees, its can reach 1.5, but at a large angle of 10 degrees -20 degrees, the value is close to 0 , indicating that there is no cubic damping term at large angle roll, it is shown that there is no cubic damping term at the rolling of large angle;Total moment of inertia of rolling $I x$, linear damping coefficient $N x$,and the squared damping $w$ have a little change With the increase of rolling angle,they remain steady all the time; The amplitude of linear restoring torque $C_{3}$ is larger, which decreases first and then increases and has minimum value when the roll angle is 10 degrees; The square recovery moment $C 2$ increases slowly,but when the draft is large,the situation is abnormal; The cubic restoring moment is maintained in a large and steady state.

\section{Summary}

In this paper, the roll decay motion in still water of the SWATH-USV is studied through model test and system identification method.Through the identification program, the mathematical model of the rolling motion which is most suitable for the ship in the 12 working conditions is found,at the same time,the roll motion process can be accurately described, and the accurate values of the torque coefficients are given.The roll motion process can be accurately described, and the accurate values of the torque coefficients are given.Meanwhile,the change rule of the moment coefficient varies with the draft and roll angle is found, and the correctness of the system identification software is verified.The research shows that the system identification software can predict the future navigation parameters and attitude of the USV and provide a reference for further studying the roll motion of the swath.

\section{References}

[1] Cunjie Liu.The research on seakeeping and longitudinal motion stability of the SWATH [D].Jiangsu university of science and technology, 2016.

[2] Qi Zhao.The research on optimized hull form and movement performance of the SWATH [D].Harbin Engineering University, 2016. 
[3] Kaiye Hu.The analysis of nonlinear rolling motion and stability of the ship in waves[D].Harbin Engineering University, 2006.

[4] X.S. Sun, C.B. Yao, Q. Ye.Numerical investigation on seakeeping performance of SWATH with Three Dimensional Translating-pulsating Source Green Function[J].Engineering Analysis with Boundary Elements.2016,73: 215-217.

[5] Shukui Liu, Apostolos Papanikolaou.Prediction of parametric rolling of ships in single frequency regular and triple frequency group waves[J].Ocean Engineering,2016,120: 274-278.

[6] Fuwei Liu, Haitong Xu, Songlin Yang.A preliminary study on roll characteristics of a amphibious USV[J].Chinese Journal of Ship Research，2014，9(1): 47-48.

[7] Tianyu Ma, Jian Cui, Songlin Yang.Maneuvering and rolling coupled mution test and analysis of the trimaran model[J].Ship Science and Technology, 2012, 34(11): 24-26.

[8] Heng Zhang, Jide Li, Xiaodong Zhao.Nonlinear motion response of ship based on system identification method[J].Journal of Dalian Maritime University, 2008，34(4): 67-71.

[9] Giulio Bottegal, Aleksandr Y. Aravkin, Hakan Hjalmarsson.Robust EM kernel-based methods for linear system identification[J].Automatica,2016,67: 114-116.

[10] Jian Lei.Research on Algorithm and application of RBF neural network optimization based on support vector machine[D].Jiangxi University of Science and Technology,2008. 